\title{
Diffusion treatment of quantum theory and gravity. 1. Diffusion quantum mechanics
}

\begin{abstract}
Zahid Zakir ${ }^{1}$
Abstract

On the basis of the observational fact that a wave packet, describing the localized ensemble of micro-objects, spreads according to the diffusion law, the quantum equivalence principle is formulated, that the motion of the ensemble of quantum objects is equivalent to the diffusion of the ensemble of classical objects in a fluctuating background field (physical vacuum). The observations also confirm the validity of the principle of relativity for quantum phenomena, formulating as the principle of constancy of quantum fluctuations, that these fluctuations and the describing them diffusion occur identically in all inertial frames. It is shown that these two physical principles, together with the principles of mechanics, lead to the diffusion quantum mechanics (DQM), containing the formalism of quantum mechanics as a particular case. In DKM the relation between the velocity of the diffusion flow and the probability density is given by the diffusion law (Fick's law), while the invariance of diffusion leads to its conservatism (diffusion without friction) and the constancy of the diffusion coefficient, fixed from correspondence with quantum mechanics. In DQM the Hamiltonian includes the kinetic energies of the drift, diffusion flux, and interaction potentials, while the probability density and the drift action function of particles $\mathrm{S}$ form a canonical pair. The canonical equations for them then lead to the continuity equation and to the Hamilton-Jacobi-Madelung equation. The probability density in them enters nonlinearly, but in the case of a canonical transformation to the complex amplitude of the probabilities, they are linearized and pass into the Schrödinger equation. As a result, the amplitudes of probabilities add up for the alternatives, i.e. their superposition takes place. DQM based on physical principles is a more general theory than quantum mechanics, since along with classical devices and particles it introduces a third participant of all processes - the background field, which is the source of quantum fluctuations of classical particles. In DQM, the quantum potential is the potential energy associated with localization, quantum statistics appears in the system of many classical particles in the fluctuating background, and composite particles of small size can have a small mass. DQM also explains the existence of rest energy, the constancy of the light velocity (velocity of quantum fluctuations) and predicts the existence and properties of gravitation as thermal diffusion in the inhomogeneous background field.
\end{abstract}

Keywords: quantum fluctuations, interpretation of quantum mechanics, non-Markovian processes, quantum statistics, principle of relativity

\section{Content}

Introduction

1. Quantum mechanics and its physical interpretation .............................................................. 4

1.1. Quantum mechanics in a canonical form ................................................................ 4

1.2. Criteria for selection of a physical mechanism ..........................................................

2. The elements of a physical mechanism of quantum mechanics .........................................5

2.1. Hydrodynamic formulation...................................................................................

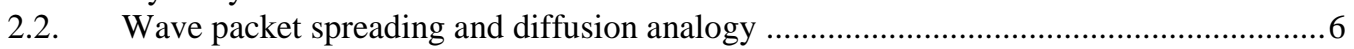

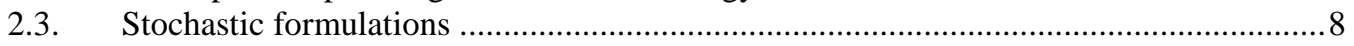

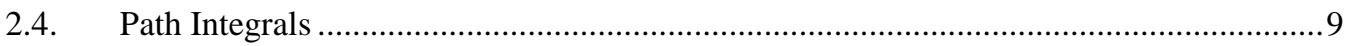

2.5. The required elements of physical mechanism in the considered treatments .................. 9

3. Diffusion quantum mechanics.................................................................................................10

${ }^{1}$ Center for Theoretical Physics and Astrophysics, Tashkent Uzbekistan, zzakir@ qgph.org 
3.1. Quantum-mechanical evolution as conservative diffusion ....................................10

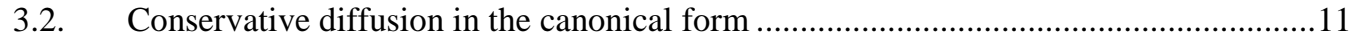

3.3. Diffusion algebra of observables leading to operator algebra.....................................13

4. Quantum statistics from conservative diffusion ...........................................................14

4.1. Quantum statistics from indistinguishability of particles .......................................14

4.2. Indistinguishability as a result of conservative diffusion ...................................... 16

5. Consequences of diffusion quantum mechanics..............................................................18

5.1. Quantum potential as the localization energy ....................................................... 18

5.2. The constancy of light velocity from the constancy of fluctuations ...............................18

5.3. The rest energy of a particle as its thermal energy in the background field .................19

5.4. Gravitation as a local deficit of fluctuation energy in the background field .................20

5.5. The scalar field condensate of SM as a candidate to the background field ...................21

5.6. Composite models with a diffusion mechanism for small mass ................................22

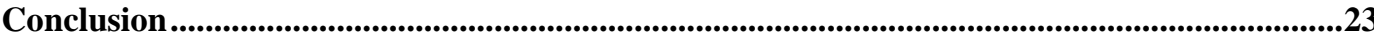

Appendix. Interpretation of quantum mechanics in DQ ........................................................23

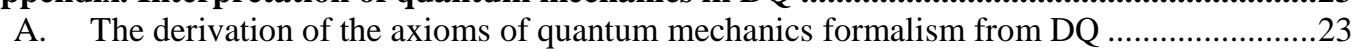

B. Interpretations of quantum mechanics: paradoxes and their solutions in DQM ................24

C. Slit experiment and the dualism of waves and particles ...........................................25

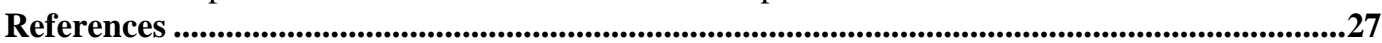

\section{Introduction}

Quantum mechanics is confirmed experimentally and forms the basis of modern physics at the level of micro-objects. However, its formalism is still based on mathematical axioms, and not on physical principles following from experiments. This means that quantum mechanics so far continued to be a very successful and reliably working mathematical model only. The incompleteness of its formation is clear from the fact that so far there was no clarity in its physical interpretation.

Therefore, the replacement of mathematical axioms with physical principles would complete the formation of quantum mechanics as a fundamental physical theory. This would lead to a clearer understanding of its physical foundations and confidence in their completeness, as well as clarify the prospects for its development and, possibly, lead to new discoveries earlier hidden in its purely mathematical form. More visibility and clearness would simplify its teaching and popularization.

The first step in this way is to find a physical mechanism leading to the formalism of quantum mechanics. But here the main difficulty was not in the absence of ideas about such mechanisms, but rather, in their too much abundance. Since the foundation of quantum mechanics, many different mechanisms have been proposed, but all without the expected result. Thus, the idea was discredited and the appearance of a new version of the mechanism began to cause more distrust than interest.

This happens when the problem is either unsolvable, or a solution has already been partially found, but turned out to be straggled in different treatments. The first case is scientifically unprovable, while the second one is quite probable and can be studied in detail. In the present paper, the problem is investigated just on the basis of the second possibility, and some of the earlier proposed physical mechanisms will be studied in their mutual connection, not as alternatives, but as complementing each other.

The first signs of the physical mechanism of quantum mechanics appeared in Madelung's attempt, when the Schrödinger equation was presented in hydrodynamic form 
[1]. In this form, the dissimilarity with classical physics was reduced to the appearance in the Hamilton-Jacobi equation of a quantum potential generated by a certain medium.

The idea of representing quantum mechanics as a theory of the motion of classical particles in a medium was supported by two more facts. Firstly, particles appear as continuously fluctuating, and the waves associated with them are probability waves, and secondly, the free localized states of their ensembles spread over time. These facts and the form of the wave equation led Ehrenfest [2], Schrödinger [3] and Fürst [4] to an analogy with some new kind of diffusion.

In particular, diffusion should have been without friction, i.e. conservative. For this reason, there were attempts in stochastic treatments $[5,6,7]$ to obtain conservative diffusion from two families of Brownian motions. But it turned out that quantum mechanics cannot be reduced to such simple Markov processes [8,9]. Nevertheless, this attempt yielded something more valuable - the formalism of conservative diffusion, which turned out to be little dependent on the particular mechanism of fluctuations.

In addition, the analogy with diffusion in its integral representation formed the basis of the physically most obvious of the standard quantization methods - the path integrals $[10,11]$, a complex version of the Wiener integral. But this method also included a number of mathematical axioms without their physical explanation.

The mathematical nature of the standard formulation and the failure of many attempts of its physical interpretation only strengthened the skepticism about the problem of the physical foundations of quantum mechanics. But partial progress, when they found elements of a physical mechanism hidden in formalism itself, showed that in reality the situation may not be so hopeless.

The problem of searching for such a mechanism then reduces to solving several particular problems, i.e. preserving those of the physical elements of previous attempts that are consistent with the formalism of quantum mechanics by eliminating contradicting to it, and then, by adding something missing, to construct from them a complete physical mechanism. Since the formalism itself was created by synthesizing different approaches, preserving their rational elements, the physical mechanism for this formalism also could be found similarly. The first aim of the paper is to solve just these problems.

The found physical mechanism will then open the way to the formulation of the physical principles of quantum mechanics, from which both its formalism and this physical mechanism itself follow, which is the second aim of the paper.

The results showed that the foundations of quantum mechanics should be completed by something new, absent in the standard formulation, - by the background field (physical vacuum), which acts as a source of the fluctuations of quantum particles. Particles themselves can be considered as classical ones, which sufficiently simplifies the theory.

The result, however, appears the formulation of a new theory, more general than quantum mechanics - diffusion quantum mechanics (DQM). As a physical theory proceeding from general physical principles resulting from experiments, DQM not only reproduces the formalism of quantum mechanics, but also gives a physical explanation for quantum phenomena, and also predicts new phenomena, in particular, thermal diffusion in the vacuum, which manifests itself as gravitation.

In this first paper, the basics of DQM are presented, and then the thermal diffusion interpretation of gravity, following from it, will be discussed in the second paper [12]. A more complete presentation of DKM will be presented in the book [13]. 
In Section 1 the problem of the physical interpretation of quantum mechanics is discussed, in Section 2 the physical mechanisms of a number of treatments related to diffusion are studied. In Section 3 and in the Appendix, the physical principles of DQM are presented and it is shown how the formalism of quantum mechanics and its treatment follow from DQM. In Sections 4 and 5 the appearance of quantum statistics is shown and the effects of DQM are discussed.

\section{Quantum mechanics and its physical interpretation}

\subsection{Quantum mechanics in a canonical form}

In quantum mechanics, the state of an object, for example a particle at a space-time point $\mathbf{x}, t$, is described by the complex probability amplitude $\psi(\mathbf{x}, t)$ related by the probability density $\rho$ as:

$$
\rho=\psi^{*} \psi, \quad \int \rho(\mathbf{x}, t) d^{3} \mathbf{x}=1,
$$

The Hamilton's function of a particle in nonrelativistic quantum mechanics has the form:

$$
H=\int\left(\frac{\hbar^{2}}{2 m} \nabla \psi^{*} \cdot \nabla \psi+\psi^{*} V \psi\right) d^{3} \mathbf{x},
$$

where the amplitudes $\psi$ and $\psi^{*}$ are a canonical pair, $\hbar=h / 2 \pi$ and $h$ is Planck constant. In the phase space of amplitudes $\left(\psi, \psi^{*}\right)$, the Poisson brackets can be defined as:

$$
\begin{gathered}
\{A, B\}=\frac{1}{i \hbar} \int\left(\frac{\delta A}{\delta \psi} \frac{\delta B}{\delta \psi^{*}}-\frac{\delta B}{\delta \psi} \frac{\delta A}{\delta \psi^{*}}\right) d^{3} \mathbf{x}, \\
\left\{\psi(x, t), \psi^{*}\left(x^{\prime}, t\right)\right\}=\delta\left(x-x^{\prime}\right) / i \hbar, \\
\left\{\psi(x, t), \psi\left(x^{\prime}, t\right)\right\}=\left\{\psi^{*}(x, t), \psi^{*}\left(x^{\prime}, t\right)\right\}=0,
\end{gathered}
$$

Then corresponding canonical equations are of the form:

$$
\begin{gathered}
\frac{\partial \psi}{\partial t}=\{\psi, H\}=\frac{1}{i \hbar} \frac{\delta H}{\delta \psi^{*}}=\frac{1}{i \hbar} \hat{H} \psi, \\
\frac{\partial \psi^{*}}{\partial t}=\left\{\psi^{*}, H\right\}=-\frac{1}{i \hbar} \frac{\delta H}{\delta \psi}=-\frac{1}{i \hbar} \hat{H} \psi^{*},
\end{gathered}
$$

and mean the standard Schrödinger equation with the Hamiltonian $\hat{H}$ :

$$
i \hbar \frac{\partial \psi}{\partial t}=\hat{H} \psi \equiv\left(-\frac{\hbar^{2}}{2 m} \Delta+V\right) \psi .
$$

The phase space $\left(\psi, \psi^{*}\right)$ is reduced to the phase space of real functions $\left(\psi_{1}, \psi_{2}\right)$, where $\psi=\psi_{1}+i \psi_{2}$. Further, the algebra of observables in the phase space $\left(\psi, \psi^{*}\right)$ leads to the operator algebra.

The linearity of the Schrödinger equation (6) leads to the fact that the superposition of amplitudes is also a solution. This property was formulated as the principle of superposition. For bound states, solutions of equation (6) lead to discrete energy levels, and 
the angular momentum and spin will also be quantized. Quantum systems differ from classical systems also by other properties, such as uncertainty relations for canonically conjugate variables and quantum statistics in a system of many particles.

\subsection{Criteria for selection of a physical mechanism}

As noted in the Introduction, we will assume that the solution to the problem of the physical interpretation of the fundamentals of quantum mechanics not only exists, but that it may be found, but distributed partially in different treatments. Our task then is to identify these elements and combine them into a joint physical mechanism.

The easiest way to do this is if we choose the most successful of the treatments, exclude from it unnecessary assumptions, and add to it those required elements which found in other treatments. At first it seems that if there was something unnecessary or contrary to quantum mechanics, then it would not have been introduced or would have been revealed and excluded over time. But this is obvious only post factum, and in practice, the presence of such elements, introduced by misunderstanding, has been interpreted as the fallacy of the entire proposed mechanism and this mechanism has been rejected. If the founders of quantum mechanics had acted like this from the beginning, the Schrödinger equation would be rejected because of Schrödinger's initial idea that a particle is a package of real waves in space.

The main distinction between the formalism of quantum mechanics and the classical one is the separation of objects into quantum and classical. Classical are objects that do not spontaneously fluctuate, while quantum objects constantly fluctuate without external sources.

At classifying the physical interpretations of quantum mechanics, we will include in the first group those that exclude such a separation. These are those where the source of quantum fluctuations is external to the objects themselves and therefore these objects themselves can remain be classical. In the second group we include those treatments where such a separation is preserved and there are objects that self-fluctuate.

First of all, we will be interested in treatments only from the first group, in which at least the first step is taken to be consistent. The treatments from the second group, by definition, are in fact the half-way solutions and we will consider them only, if the first group appears as unsuccessful.

In the next section, we consider a number of treatments with searched elements of the physical mechanism of quantum mechanics. They are unified by an attempt to formulate quantum phenomena in classical terms, in particular, on the basis of classical particles moving along continuous trajectories, even though irregular.

\section{The elements of a physical mechanism of quantum mechanics}

\subsection{Hydrodynamic formulation}

According to (1), the expression $\psi^{*} \psi$ is the probability density $\rho$ and, therefore, any complex valued amplitude can be written through the module $\rho$ and phase $S / \hbar$ [1]:

$$
\psi=\sqrt{\rho} \exp (i S / \hbar) .
$$

The Schrödinger equation (6) then appears as the equations for real valued functions $\rho, S$ : 


$$
\begin{gathered}
\frac{\partial S}{\partial t}+\left(\frac{(\nabla S)^{2}}{2 m}+V\right)-\frac{\hbar^{2}}{2 m} \frac{\Delta \sqrt{\rho}}{\sqrt{\rho}}=0, \\
\frac{\partial \rho}{\partial t}+\nabla(\rho \cdot \nabla S / m)=0 .
\end{gathered}
$$

The second Eq. (9) is the continuity equation for the flux density $\rho \mathbf{v}$ with the drift velocity

$$
\mathbf{v}=\nabla S / m,
$$

which shows that $S(\mathbf{x}, t)$, the phase of the amplitude, is an analog of the action function along the classical trajectory with this drift velocity.

The first equation (8), the Hamilton-Jacobi-Madelung equation contains, in addition to the classical terms, also a "quantum potential" $U_{q}$ :

$$
U_{q}=-\frac{\hbar^{2}}{2 m} \frac{\Delta \sqrt{\rho}}{\sqrt{\rho}} .
$$

Eqs. (7)-(10) form the hydrodynamic formulation of quantum mechanics, and many attempts to search for the physical mechanism of the theory started from them. The theory is formulated in terms of two functions, $S$ and $\rho$ having a more or less clear classical meaning, and everything related to the mechanisms of quantum fluctuations is reduced to the appearance of $U_{q}$ in the Hamilton-Jacobi equation.

Thus, the problems of physical interpretation of quantum mechanics are reduced to answer to two questions - why particles are described by the probability density $\rho$ and where does the quantum potential $U_{q}$ come from.

The hydrodynamic formulation itself, of course, cannot answer them, which is the half way nature of all such formal approaches. But the very fact of the representation of quantum mechanics in terms of purely classical concepts and equations is exciting and promising, stimulating a search in the already outlined direction.

\subsection{Wave packet spreading and diffusion analogy}

After the discovery of the Schrödinger equation, the author's hypothesis that it describes real waves in space, and that a particle is a wave packet, did not justified for two physical reasons. These are the proof of the probabilistic nature of the wave function and the wave packet spreading.

These two properties of quantum-mechanical motion inspired of P. Ehrenfest to an analogy with the heat equation [2], and then of Schrödinger [3] and Fürst [4] to an analogy with diffusion described by the same equation. The spreading of the states of a free quantum particle, initially localized in a small region, turned out to be similar to the diffusion of a classical particle in a fluctuating medium. Since the drift velocity $\mathbf{v}$ of the ensemble of free quantum particles is constant, the corresponding diffusion should have been without friction, i.e. conservative.

The Schrödinger equation (6) for a free particle, written in the form:

$$
\frac{\partial \psi}{\partial t}=\bar{D} \Delta \psi, \quad \bar{D}=i D, \quad D=\frac{\hbar}{2 m},
$$


similar to the classical diffusion equation:

$$
\frac{\partial \rho}{\partial t}=D_{c} \Delta \rho
$$

Therefore, although in (12) there is $\rho$ instead of the amplitude $\psi$, and the diffusion coefficient $\bar{D}$ is imaginary, nevertheless, the solution for $\psi$ is the same as for $\rho$, but with the replacement $D_{c} \rightarrow \bar{D}$. In particular, in the one-dimensional case it has form [2]:

$$
\psi(x, t)=\int_{-\infty}^{+\infty} \frac{d x_{0}}{2 \sqrt{i \pi \hbar t / m}} e^{-\frac{\left(x-x_{0}\right)^{2}}{2 i \hbar t / m}} \psi\left(x_{0}, 0\right) .
$$

Let the wave packet be constructed so that at the moment $t=0$ the probability of detecting a free particle is large only in a small neighborhood $\pm a$ of the point $x_{0}=0$ and that the centre of the packet moves with the velocity $v_{0}$ :

$$
\psi\left(x_{0}, 0\right)=C e^{-\frac{x_{0}^{2}}{2 a^{2}}+i \frac{p_{0} x_{0}}{\hbar}}, \quad \rho\left(x_{0}, 0\right)=C^{2} e^{-\frac{x_{0}^{2}}{a^{2}}},
$$

where $p_{0}=m v_{0}$. Substituting this into (14), we find the probability density:

$$
\rho(x, t)=\frac{A^{2}}{\left[1+\left(\hbar t / m a^{2}\right)^{2}\right]} e^{-\frac{\left(x-v_{0} t\right)^{2}}{a^{2}\left[1+\left(\hbar t / m a^{2}\right)^{2}\right]}} .
$$

This is a well-known formula for the wave packet spreading, according to which the values of $\rho$ decrease everywhere and the width of the packet grows in time. As a result, the ensemble of particles uniformly decomposes in the space and the probability of finding a particle from this ensemble will become almost the same everywhere.

The most important property of formula (16) is that it includes the square of the modulus of the "diffusion coefficient" $|\bar{D}|^{2}$ from (12) and therefore the "spreading" $\rho$ with time proceeds as in the case of ordinary diffusion in the rest frame of the packet's center, although faster. The fact that a probability is included $D t / a^{2}$ in the probability density of ordinary diffusion, but in the quantum mechanical one a square of this quantity $\left(D t / a^{2}\right)^{2}$, does not change the diffusion nature of the process. This only indicates that this diffusion, being conservative, occurs faster than usual, which is why spreading is faster.

A fact of fundamental importance is that, from a physical point of view, quantum fluctuations appear as a diffusion process, although a specific one. But, despite this specificity, there is mainly similar to the ordinary diffusion, the consequences of which will be further studied in more detail.

In any diffusion, along with the drift flux $\rho \mathbf{v}$ from (10), there will be also a diffusion flux $\rho \mathbf{u}$ spreading the probability density $\rho$. This flow in a homogeneous classical medium obeys the diffusion law (Fick's law):

$$
\mathbf{u}_{c} \rho=-D_{c} \nabla \rho .
$$


If the evolution of a quantum particle is similar to diffusion and $\rho$ has the same form as for diffusion, then the diffusion law (17) should be applicable to $\rho$ also. In this case, as in (16), it should include not $\bar{D}$, but its module $D=|\bar{D}|=\hbar / 2 m$ :

$$
\mathbf{j}_{D} \equiv \mathbf{u} \rho=-\frac{\hbar}{2 m} \nabla \rho .
$$

This gives an expression for the diffusion velocity $\mathbf{u}$ :

$$
\begin{gathered}
\mathbf{u}=-\frac{\hbar}{2 m} \frac{\nabla \rho}{\rho} . \\
\overline{\mathbf{u}}=\int \mathbf{u} \rho d^{3} \mathbf{x}=0, \quad \int \mathbf{u}^{2} \rho d^{3} \mathbf{x} \neq 0 .
\end{gathered}
$$

In addition, from the relations:

$$
\overline{\mathbf{p}_{u}^{2}} \cdot \overline{\mathbf{x}^{2}} \geq\left|\overline{\mathbf{p}_{u} \cdot \mathbf{x}}\right|^{2}=m^{2}\left|\int \mathbf{u} \cdot \mathbf{x} \rho d^{3} \mathbf{x}\right|^{2}=(m D)^{2}\left|\int \nabla \rho \cdot \mathbf{x} d^{3} \mathbf{x}\right|^{2}=\frac{\hbar^{2}}{4} .
$$

where $\overline{\mathbf{x}}=0$, it follows that for $\mathbf{p}_{u}=m \mathbf{u}$ there is the uncertainty relation. In the diffusion picture, this is explained by the fact that the diffusion flux in a homogeneous medium tends to reduce the degree of localization of the ensemble of particles [2-4]. As smaller the localization region, as greater the gradient of probability density $\nabla \rho$ and as greater the diffusion velocity $\mathbf{u}$ directed backward to this gradient.

Thus, the analogy with diffusion provides answers to both questions of the hydrodynamic picture. At first, the particles diffuse in a fluctuating medium, and therefore the ensemble of particles is described by a probability density $\rho$. Secondly, the Hamiltonian of the ensemble of particles includes the kinetic energy of the diffusion flux $\mathbf{p}_{\mathbf{u}}^{2} / 2 m$ leading to the quantum potential $U_{q}$ (11), earlier seemed mysterious.

So, the spreading over time of the initially localized ensemble of particles in the formalism of quantum mechanics is similar to the diffusion process, which gives a physical explanation for both the quantum potential $U_{q}$ and the uncertainty relation.

\subsection{Stochastic formulations}

The two considered above treatments, based on hydrodynamic and diffusion analogies, are completely equivalent from the mathematical point of view to the standard operator formalism of quantum mechanics. In contrast, stochastic treatments introduce what is absent in the formalism of quantum mechanics - a family of Brownian processes in a fluctuating background field, which should have led to the conservative diffusion.

The most detailed investigated of these treatments is stochastic mechanics [5,6,7], in which conservative diffusion arises from the combination of two families of Brownian processes going from the past and going into the future.

However, it turned out that the conditional probabilities, or transition probabilities, required for determining the velocities and root-mean-square displacements of particles cannot be identified with transition probabilities known from the theory of Brownian motion $[8,9]$. Thus, quantum mechanics could not be reduced to such simple Markov processes, and, as a result, the whole approach was considered erroneous. 
But, although the initial hypotheses of stochastic mechanics did not give the expected result, nevertheless, within its framework, a formalism of conservative diffusion was created. It has a more universal character than particular assumptions about fluctuations on the basis of which it was discovered. Therefore, it remains valid in DQM, further developed in the present paper, in which, unlike stochastic mechanics, there is no need for a hypothesis about the Markov nature of fluctuations.

\subsection{Path Integrals}

Brownian motion of a particle in a medium with friction leads to dissipative diffusion, which is usually described by differential equations. But this diffusion can also be described through the Wiener path integral. Similarly, random walks of a frictionless particle leading to conservative diffusion can be described both by the Schrödinger equation, a complex analog of the diffusion equation, and the Feynman integral [10, 11], a complex analog of the Wiener integral.

Being the most physical of the frequently used quantization methods, quantization in terms of path integrals is still only partially physical. It contains several mathematical axioms, such as recipes for replacing the probability adding rules to the probability amplitudes adding rules, as well as the relationship of these amplitudes with the action function for particles along their classical, but random trajectories. Until now, these axioms had no physical explanation and therefore the method remained be formal-mathematical.

Nevertheless, the achievement of this treatment is the fact that the transition amplitudes are formulated completely in terms of classical concepts, such as trajectories and the action function along them. The distinction from classical physics was reduced only to the fact that there is a summation: a) not the probabilities, but the amplitudes of the transition probabilities, and b) not along the extreme but along all possible trajectories, and also c) according to a certain relation between the amplitude and the action function.

Just as in the hydrodynamic formulation, the key question about the source of fluctuations remains open - whether the particles themselves fluctuate, or some external source makes them fluctuate. The distinction is that the question of the quantum potential is replaced by two other questions - the origin of the amplitudes addition rules and the relation of the amplitudes and the action function.

\subsection{The required elements of physical mechanism in the considered treatments}

Four treatments were briefly considered above, which form the basis of the first group of treatments, where the particles remain be classical and fluctuate due to interaction with a fluctuating background. In the diffusion and stochastic treatments, this lies at their basis, and in the hydrodynamic treatment and the path integrals, this is implicitly assumed.

In the standard operator formalism of quantum mechanics, two elements remain classical - this is space-time, as well as measuring devices. This is true in all four considered treatments from the first group. Therefore, the first two elements of the joint mechanism that we are looking for, it is natural to take them, namely, the classic space-time and measuring devices.

As the following two elements, it is natural to take what is common to these four interpretations - firstly, the classical nature of objects, in particular particles, and secondly, the hypothesis of the existence of a fluctuating background field, which is an external source of fluctuations of these particles. 
After determining the source of fluctuations, it is further necessary to reveal the properties and consequences of these fluctuations. Here we take into account the fact that the simple Brownian processes with the Markov property do not solve our problem. The theory of non-Markov processes also did not help to construct the correct replacement for stochastic mechanics, since it further complicated already difficult situation.

However, it will be shown below that the equivalence of spreading of the probability density to the wave packet to a certain diffusion process gives almost everything that is required to reproduce the formalism of quantum mechanics. This property can be called the "quantum principle of equivalence", since it allows us to reduce the description of complicated quantum systems to the description of diffusion processes, the theory of which is well known and quite simple.

Therefore, further the diffusion process, albeit conservative, will become the basic phenomenon for the desired physical mechanism of quantum mechanics.

Thus, the initial observational fact on which the physical interpretation of quantum mechanics can be based is the above-mentioned "quantum principle of equivalence", which allows us to reduce quantum mechanics to the theory of diffusion of classical particles in a fluctuating background field.

Notice that in particle physics there is a scalar field which gives masses to particles, and it can be expected that fluctuations of the classical component of this field could also generate quantum fluctuations of particles (see Section 5.5).

\section{Diffusion quantum mechanics}

\subsection{Quantum-mechanical evolution as conservative diffusion}

If the behavior of an ensemble of quantum particles is similar to the specific diffusion of classical particles, then it is necessary to clear up in what this specificity consists of and how this type of diffusion is described.

In ordinary diffusion of a particle in a condensed medium, friction plays an essential role. In the simplest case, when this medium is homogeneous, isotropic, and rests in one of the inertial frames $K$, the diffusion of an ensemble of particles resting on average in $K$ is described by diffusion equation (13) with $D_{c}=$ const .

If at first the ensemble of particles has a non-zero drift velocity $\mathbf{v}(0) \neq 0$ with respect to $K$ and rests in another inertial frame $K^{\prime}$, then due to friction, i.e. resistance of the medium, this drift velocity will decrease and disappear with time. For the observer in $K^{\prime}$, this looks like the appearance of a drift velocity of earlier resting particles due to their entrainment by a moving medium, until they reach the comoving velocity to this medium.

Ordinary diffusion is thus dissipative, and the rest frame of the environment is the preferred frame of reference for the diffusing ensemble.

At the same time, all the experiments where quantum phenomena were studied did not reveal any preferred reference frame for them. On the contrary, in all inertial frames, quantum phenomena occur similarly in exact agreement with the principle of relativity. This means that the main specificity of quantum fluctuations and their corresponding diffusion is that they satisfy the principle of relativity and occur similarly in all inertial frames.

We formulate this experimental fact as one of the physical principles of DQM, as the principle of the constancy of quantum fluctuations and diffusion. In accordance with 
this principle, fluctuations of classical particles due to interaction with the background field and the diffusion generated by them are the same in all inertial frames.

In particular, if at first the probability density for the ensemble of free particles in $K$ had a Gaussian form (15), then in $K^{\prime}$ the center of this distribution will move with a constant drift velocity. Conversely, an ensemble with the same distribution, but with the center resting at $K^{\prime}$, diffuses in $K^{\prime}$ exactly the same as in $K$, but now it will have a constant drift velocity with respect to $K$. Thus, the desired specificity of diffusion is that it should be without friction, i.e. it is conservative diffusion.

In addition, from the homogeneity and isotropy of space and time, it follows that the diffusion coefficient of such a conservative diffusion is constant. Below, from the correspondence with quantum mechanics, it will be fixed as equal to $D=\hbar / 2 m$.

Thus, to construct the theory of quantum phenomena, we need to consider the description of the conservative diffusion of classical particles in a fluctuating background field with the diffusion coefficient $D$. The existing observations and the physical principles based on them, in particular, the principle of constancy of quantum fluctuations and diffusion, do not allow us to clarify the nature and source of quantum fluctuations.

Nevertheless, already at this stage of our knowledge, the hydrodynamic picture, in which the diffusion processes are described in terms of densities and flows, without specifying the microscopic mechanism of fluctuations generating these flows, turns out to be a powerful method allowing us to describe and explain quite a lot of phenomena.

\subsection{Conservative diffusion in the canonical form}

The diffusion of an ensemble of particles is described by the probability density $\rho(\mathbf{x}, t)$ normalized to unity (1). The diffusion law (Fick's law):

$$
\mathbf{j}_{D} \equiv \mathbf{u} \rho=-\frac{\hbar}{2 m} \nabla \rho
$$

gives for the local diffusion velocity $\mathbf{u}$ in the ensemble the expression:

$$
\mathbf{u}=-\frac{\hbar}{2 m} \frac{\nabla \rho}{\rho} .
$$

Any diffusion of an ensemble of particles is also characterized by a drift velocity $\mathbf{v}(\mathbf{x}, t)$, which is equal to the sum of the initial mean velocity (in a given inertial frame) and the velocity appeared due to acceleration in an external potential $V$. But in the case of the conservative diffusion in a homogeneous and isotropic space, due to the absence of friction, $\mathbf{v}$ is similar to usual mean velocity in mechanics. Therefore, the drift momentum $\mathbf{p}_{\mathbf{v}}$, as well as in classical mechanics, corresponds to the drift action function $S$ and therefore $\mathbf{v}$ is expressed in the same way as in (10):

$$
\mathbf{p}_{\mathbf{v}}=m \mathbf{v}=\nabla S, \quad \mathbf{v}=\nabla S / m .
$$

The Hamilton's function $H_{0}$ of the ensemble of free particles contains the kinetic energy of the drift $\mathbf{p}_{\mathbf{v}}^{2} / 2 m$ and the kinetic energy of the diffusion flux $\mathbf{p}_{\mathbf{u}}^{2} / 2 m$. Adding to $H_{0}$ the potential $V$ of external forces, we obtain the complete Hamilton's function: 


$$
H=\int\left(\frac{\mathbf{p}_{\mathbf{v}}^{2}}{2 m}+\frac{\mathbf{p}_{\mathbf{u}}^{2}}{2 m}+V\right) \rho d^{3} \mathbf{x},
$$

which, taking into account (23) and (24), takes the form:

$$
H=\int\left(\frac{1}{2 m}(\nabla S)^{2}+\frac{\hbar^{2}}{8 m}\left(\frac{\nabla \rho}{\rho}\right)^{2}+V\right) \rho d^{3} \mathbf{x} .
$$

To describe the motion of a classical particle in empty space, its coordinates $\mathbf{x}(t)$ and momenta $\mathbf{p}=m \dot{\mathbf{x}}$ are required. In the Hamilton's function $H$ (26), the role of the generalized coordinate is played by the function $\rho$ as a certain field variable. But only $\rho$ and its spatial derivatives given at the moment $t$ enter into $H$, while the time derivative $\partial_{t} \rho$ does not appear. Therefore, the usual canonical formalism of field theory is not directly applicable here and some modification is required.

For this purpose, we pay attention to the fact that, the Hamilton's function (26) includes, in addition to $\rho$, only the drift action function $S$ from (24) related by the drift velocity $\mathbf{v}$. Thus, of the two functions, $\rho$ and $S$, only $S$ turns out to be related by the drift velocity $\mathbf{v}$, a variable connecting two moments of time. Therefore, we can identify $S$ with the generalized momentum canonically conjugate to $\rho$.

The canonical pair $\rho$ and $S$ then determines the Poisson brackets in the phase space $(\rho, S)[7]:$

$$
\begin{gathered}
\{A, B\}=\int\left(\frac{\delta A}{\delta \rho} \frac{\delta B}{\delta S}-\frac{\delta B}{\delta \rho} \frac{\delta A}{\delta S}\right) d^{3} \mathbf{x}, \\
\left\{\rho(x, t), S\left(x^{\prime}, t\right)\right\}=\delta\left(x-x^{\prime}\right), \\
\left\{\rho(x, t), \rho\left(x^{\prime}, t\right)\right\}=\left\{S(x, t), S\left(x^{\prime}, t\right)\right\}=0 .
\end{gathered}
$$

The canonical equations in this case take the form:

$$
\frac{\partial S}{\partial t}=\{S, H\}=-\frac{\delta H}{\delta \rho}, \quad \frac{\partial \rho}{\partial t}=\{\rho, H\}=\frac{\delta H}{\delta S} .
$$

Substituting into (29) the Hamilton's function $H$ from (26) and transforming with the addition of the full derivative:

$$
\left(\frac{\nabla \rho}{\rho}\right)^{2}+2 \nabla\left(\frac{\nabla \rho}{\rho}\right)=4 \frac{\Delta \sqrt{\rho}}{\sqrt{\rho}},
$$

we can rewrite the canonical equations (29) in the form:

$$
\begin{gathered}
\frac{\partial S}{\partial t}+\left(\frac{(\nabla S)^{2}}{2 m}+V\right)-\frac{\hbar^{2}}{2 m} \frac{\Delta \sqrt{\rho}}{\sqrt{\rho}}=0, \\
\frac{\partial \rho}{\partial t}+\nabla(\rho \cdot \nabla S / m)=0,
\end{gathered}
$$


These are nothing but as the well-known Hamilton-Jacobi-Madelung equation (8) and the continuity equation (9). Their linearization by canonical transformation:

$$
\psi=\sqrt{\rho} e^{i S / \hbar},
$$

gives a transition from $(\rho, S)$ to the phase space $\left(\psi^{*}, \psi\right)$ formed by the of probability amplitude, and two real-valued canonical equations (31)-(32) form one complex equation - the Schrödinger equation:

$$
i \hbar \frac{\partial \psi}{\partial t}=-\frac{\hbar^{2}}{2 m} \Delta \psi+V \psi
$$

Since one of the canonical equations is the continuity equation, where $\partial_{t} \rho$ is determined, except $\rho$, only by $S$, more precisely $\nabla S$, this confirms the correctness of our choice of $S$ as the canonical momentum, since it is this function that represents $\partial_{t} \rho$ in the Hamiltonian when the canonical equations are satisfied.

Notice that the canonical transformations in $(\rho, S)$, preserving the normalization conditions (1), correspond to the unitary transformations in $\left(\psi^{*}, \psi\right)$.

Thus, the conservative diffusion of classical particles in the background field with the diffusion coefficient $D=\hbar / 2 m$ and the motion of quantum particles in accordance with the formalism of quantum mechanics are equivalent.

This fact, which directly follows from experiments on the passage of particles through a slit on the screen [11], is formulated as the second physical principle of DQM and will be called the quantum principle of equivalence. According to this principle, the evolution in time of the probability density of an initially localized ensemble of free quantum particles is equivalent to the evolution of the probability density of an ensemble of classical particles during their conservative diffusion in the fluctuating medium (background field) with the diffusion coefficient $D=\hbar / 2 m$.

Therefore, classical mechanics, supplemented by two principles, the principle of constancy of quantum fluctuations and diffusion, and the quantum principle of equivalence, allows us to naturally derive the formalism of quantum mechanics.

In this case, quantum mechanics turns out to be a special case of a more general theory, the theory of conservative diffusion of classical particles in the fluctuating background field, which can be called diffusion mechanics (DM). However, given the fact that the choice $D=\hbar / 2 m$ relates specifically to the case of quantum mechanics, the name diffusion quantum mechanics (DKM) is more appropriate, which we will use further.

\subsection{Diffusion algebra of observables leading to operator algebra}

In the theory of conservative diffusion, the observable $A$ is also a function of $\rho$ and $S$ in the phase space $(\rho, S)$. In the corresponding phase space $\left(\psi^{*}, \psi\right)$, it corresponds to the bilinear form of the function $A\left(x, x^{\prime}\right)=A\left(x^{\prime}, x\right)$ [7]:

$$
A(\rho, S)=\iint \psi^{*}(x) A\left(x, x^{\prime}\right) \psi\left(x^{\prime}\right) d x d x^{\prime} .
$$


The observables are real-valued and therefore it is possible to introduce a Hermitian operator $\hat{A}$ acting on the amplitude as:

$$
(\hat{A} \psi)(x)=\int A\left(x, x^{\prime}\right) \psi\left(x^{\prime}\right) d^{3} \mathbf{x}^{\prime} .
$$

Then the bilinear form (35) takes the usual form:

$$
A(\rho, S)=\int \psi^{*}(x) \hat{A} \psi(x) d^{3} \mathbf{x} \equiv\langle\psi, \hat{A} \psi\rangle .
$$

In particular, the Poisson bracket of two observables (27) takes the form of a bilinear form from the commutator of two operators corresponding to them:

$$
\{A, B\}=(1 / i \hbar)\langle\psi,[\hat{A}, \hat{B}] \psi\rangle
$$

The mean value of the particle's coordinate along the trajectory as observable is:

$$
\overline{\mathbf{x}}(t)=\int \rho \mathbf{x} d^{3} \mathbf{x}=\langle\psi, \mathbf{x} \psi\rangle .
$$

Invariance with respect to translation $\mathbf{x}^{\prime}=\mathbf{x}+\mathbf{a}$ leads to conservation of momentum, for which there is a standard expression in terms of operators:

$$
\overline{\mathbf{p}}=\langle\psi, \hat{\mathbf{p}} \psi\rangle, \hat{\mathbf{p}}=(\hbar / i) \nabla
$$

In the diffusion picture, this observable has the form:

$$
\overline{\mathbf{p}}(\rho, S)=\int \rho(m \mathbf{v}+i m \mathbf{u}) d^{3} \mathbf{x}=\int \rho \nabla S d^{3} \mathbf{x}
$$

and is a shift generator for the canonical pair:

$$
\begin{gathered}
\delta \rho=\delta \mathbf{a} \cdot\{\rho, \overline{\mathbf{p}}\}=\delta \mathbf{a} \cdot \frac{\delta \overline{\mathbf{p}}}{\delta S}=-\delta \mathbf{a} \cdot \nabla \rho, \\
\delta S=\delta \mathbf{a} \cdot\{S, \overline{\mathbf{p}}\}=-\delta \mathbf{a} \cdot \frac{\delta \overline{\mathbf{p}}}{\delta \rho}=-\delta \mathbf{a} \cdot \nabla S .
\end{gathered}
$$

The time derivative of the observable is also similar to the operator definition:

$$
\frac{d A}{d t}=\frac{\partial A}{\partial t}+\{A, H\}=\frac{\partial A}{\partial t}+\frac{1}{i \hbar}\langle\psi,[\hat{A}, \hat{H}] \psi\rangle
$$

\section{Quantum statistics from conservative diffusion}

\subsection{Quantum statistics from indistinguishability of particles}

Above a diffusion description of a state with a single particle has been studied. The ensemble of experiments allowed us to determine the probabilities and consisted on a set of experiments by one a particle in any case under the same conditions.

In statistical mechanics, systems of a very large number of particles are considered, where the source of fluctuations is the randomness of collisions or other forms of mutual interactions in this multiparticle system. The phase space of a system of particles is divided into small cells and the energy intervals of particles in these cells are studied. In particular, to determine $P\left\{n_{i}\right\}$, the probabilities of filling $M$ states with filling numbers $\left\{n_{i}\right\}, i=1, \ldots, M$, we need to know the number of ways (statistical weight) $\Delta \Gamma$ by which these states can be filled [14]. 
In classical statistical mechanics, firstly, particles are considered as distinguishable, and secondly, the probabilities of all states are assumed to be identical, i.e. are considered equal $w_{i}=w=1 / M$. In classical statistics, the number of states is very large and therefore the average occupancy numbers of states are small compared to unity, i.e. although the number of particles $N$ is large, it is still small in comparison with the number of states $M$, i.e. $N \ll M$. For small average occupancy numbers, particles are distributed over states independently of each other.

By placing each of the $N$ various particles in one of the $M$ states, we obtain $M^{N} n_{1} ! n_{2} ! \ldots n_{M}$ ! possible distributions. Since those of them that differ only in the permutation of the particles are considered as identical ones, we must also divide this number to the number of permutations $N$ !. Thus, we find the statistical weight $\Delta \Gamma_{M B}$ of the distribution of $N$ particles over $M$ states, the inverse of which is $P_{M B}\left\{n_{i}\right\}$ :

$$
P_{M B}\left\{n_{i}\right\}=\frac{1}{\Delta \Gamma_{M B}}=\frac{M^{-N} N !}{n_{1} ! n_{2} ! \ldots n_{M} !} .
$$

From the requirement that the entropy $S=\ln \Delta \Gamma$ be maximal at equilibrium, then the distribution function of the particles of the equilibrium gas is found in classical statistics (Maxwell-Boltzmann distribution (MB)):

$$
\bar{n}_{i}=e^{-E_{i} / k T} \text {. }
$$

In the formalism of quantum mechanics, the probabilities of realization of different states of a system of particles were also considered similarly and the distinction from classical statistics was reduced to two properties: a) the particles were considered as indistinguishable, and b) the occupation numbers did not assume to be small.

It turned out that the indistinguishability property changes the statistical weight of states in such a way that instead of classical statistical mechanics one of the two forms of quantum statistics appears [14]. The first of them relates to a system of particles with a wave function symmetric under permutations of pairs of particles and in each state, as in the classical case, there can be any number of particles (Bose-Einstein statistics (BE)). The second relates to the systems where the amplitude is antisymmetric and therefore no more than one particle can be in each state (Fermi-Dirac statistics (FD)).

In BE statistics, under the assumption of indistinguishability of particles, each of the $\left\{n_{i}\right\}$ configurations defines one state. Therefore, the statistical weight of states $\Delta \Gamma$ is the number of ways in which $N$ identical particles can be placed over $M$ states. The number of such placements, or statistical weight $\Delta \Gamma_{B E}$, is well known from combinatorics and for the filling probability, which is the inverse of this number, we obtain an expression that is no longer dependent on $\left\{n_{i}\right\}$ :

$$
P_{B E}\left\{n_{i}\right\}=\frac{1}{\Delta \Gamma_{B E}}=\frac{N !(M-1) !}{(M+N-1) !} .
$$

Together with the maximum entropy condition, this gives the BE distribution:

$$
\bar{n}_{i}=\frac{1}{e^{E_{i} / k T}-1} .
$$

In the second case, in FD statistics, the statistical weight of states $\Delta \Gamma_{F D}$ is the number of ways in which $N$ identical particles can be selected from $M$ states. In 
combinatorics, this is known as the number of combinations of $M$ elements in $N$ and its inverse gives the desired probability $P_{F D}\left\{n_{i}\right\}$ :

$$
P_{F D}\left\{n_{i}\right\}=\frac{1}{\Delta \Gamma_{F D}}=\frac{N !(M-N) !}{M !} .
$$

Together with the maximum entropy condition, this gives the FD distribution:

$$
\bar{n}_{i}=\frac{1}{e^{E_{i} / k T}+1} .
$$

Thus, it is exactly the assumption of quantum mechanics about the indistinguishability of particles leading to the appearance of quantum statistics for two kinds of states, depending on whether their probability amplitude is symmetric or antisymmetric at permutations of pairs of particles.

\subsection{Indistinguishability as a result of conservative diffusion}

In DQM particles remain classical and therefore remain distinguishable. Therefore, a non-trivial question arises: how can quantum statistics, in which particles are indistinguishable, appear in a system of classical distinguishable particles?

There are two types of probabilistic laws in a gas of microparticles - collisions between particles lead to statistical laws similar to classical statistics, and conservative diffusion of particles between collisions lead to quantum properties of the system. The role of the diffusion between collisions is insignificant at high temperatures and a high density of states, when the filling numbers are small, but becomes significant at low temperatures and not small filling numbers.

Therefore, if for classical particles in empty space all the states are equally probable, then for the same particles that also fluctuate between collisions due to the background field, these probabilities do not have to be equal.

Most easily, this distinction is manifested for states with antisymmetric wave functions, when there can be no more than one particle in one state. This property is not related to the multiparticle system and holds even for a system of two particles, i.e. this is a consequence of purely quantum fluctuations. Therefore, for such systems, instead of the number of distributions $M^{N}$, it is necessary to take only the number of combinations of $M$ elements along $N$ and instead of (45) we obtain (49), as a result of which the MB distribution (46) is replaced by the FD distribution (50).

The reason why in the system of classical particles appears BE statistics was found by Terzoff and Bayer [15]. It turned out that the equal probability condition of different states of distinguishable classical particles, i.e. when the probability of a particle falling into each of the $M$ states is equal $1 / M$, is the unnecessarily strong restriction. Introducing a weaker condition instead, they obtained the BE distribution in classical physics.

The weakening of the conditions meant that in the general case these probabilities can take arbitrary values $w_{1}, \ldots, w_{M}$ limited only by the usual properties of probabilities $0 \leq w_{i} \leq 1, \sum_{i=1}^{M} w_{i}=1$, and it is enough to average over all their admissible values. This reduces to the integrations with respect to $w_{i}$, limited only by the fact that the sum of the probabilities should be equal to unity: 


$$
\frac{\left(\prod_{i=1}^{M} \int_{0}^{1} d w_{i} \cdot w_{i}^{n_{i}}\right) \delta\left(1-\sum_{i=1}^{M} w_{i}\right)}{\left(\prod_{i=1}^{M} \int_{0}^{1} d w_{i}\right) \delta\left(1-\sum_{i=1}^{M} w_{i}\right)}=\left(\prod_{i=1}^{M} \int_{0}^{1} d w_{i} \cdot w_{i}^{n_{i}}\right) \delta\left(1-\sum_{i=1}^{M} w_{i}\right)
$$

There is a question about why equiprobability should be replaced by the averaging over arbitrary probabilities. There was no answer to this in [15], because there is no reason for this in the classical ideal gas, while in standard quantum mechanics the particles are not classical.

However, in the framework of DQM, there is a clear answer to this question. Classical gas dynamics assumes that between collisions the particles move rectilinearly in empty space. But in DQM, classical particles interact with the background field also and, for this reason, make additional fluctuations between collisions. Therefore, for each particle, the filling probabilities attain additional random deviations. This implies the need to account these deviations of probabilities by averaging over all their values as in (51).

Thus, for distinguishable particles, we replace the assumption of equal probability of states with the assumption that different configurations have different probability weights, i.e. we assign to each discrete state $i$ an arbitrary probability of weight $\left\{w_{i}\right\}$ with usual restriction on probability. As a result, the probability of the MB statistics (45) should be replaced by:

$$
P\left\{n_{i}\right\}=N !\left[\prod_{i=1}^{M} \frac{1}{n_{i} !} \int_{0}^{1} d w_{i} \cdot w_{i}^{n_{i}}\right] \delta\left(1-\sum_{i=1}^{M} w_{i}\right) .
$$

Integrals are calculated by including constraints on $w_{i}$ into the limits of integrations, i.e. taking into account $0 \leq w_{2} \leq 1-w_{1}$ at $M=2$ etc. Each following integral has the form

$$
\int_{0}^{a} d w \cdot w^{m}(a-w)^{n}
$$

and is calculated recursively, which ultimately gives the result of BE (47).

Thus, in the gas of distinguishable particles, which between collisions do not move along classical trajectories, but also additionally fluctuate, there is a BE distribution. The correlation between particles in different states, which is absent in classical mechanics, in DQM arises from the fact that diffusion of particles is described by probability amplitudes defined globally, and the amplitudes of many particles mutually overlap. The indistinguishability of particles in quantum statistics manifests itself effectively as a consequence of taking into account these additional fluctuations and the associated with them correlations between particles.

A fundamentally new property of quantum fluctuations is the Pauli exclusion principle for systems of particles described by the wave function antisymmetric under the permutation of particles. By no discussing the details of the connection of the relationship between spin and statistics, we only note that DQM, which describes processes by means the probability amplitudes, generally admits such states regardless of the reasons for their existence. The probability density $\rho$ is the square of the amplitude modulus $|\psi|^{2}$ and the amplitude, in contrast to the probability, can has both signs:

$$
\psi= \pm|\psi| \text {. }
$$


Therefore, if for some reason there is conservative diffusion with antisymmetric wave functions, changing the sign at the permutations of particles, then all consequences of the Fermi-Dirac statistics and the Pauli principle will be observed, following from a change in the sign of the wave function at such permutations.

\section{Consequences of diffusion quantum mechanics}

\subsection{Quantum potential as the localization energy}

A classical particle in a classical empty space and external potential $V$ would have the total energy $\mathbf{p}_{\mathbf{v}}^{2} / 2 m+V$. An ensemble of such particles in the physical space moves in the background field and therefore makes quantum fluctuations. Moreover, if the probability density has an initial heterogeneity, then a diffusion flux $\rho \mathbf{u}$ arises. Let us consider the physical meaning of the energy of this diffusion flow $\bar{U}_{u}$ :

$$
\bar{U}_{u}=\int \frac{\mathbf{p}_{\mathbf{u}}^{2}}{2 m} \rho d^{3} \mathbf{x} .
$$

The state of a free particle is a plane wave:

$$
\psi=\text { const } \cdot \mathrm{e}^{i(\mathbf{p x}-E t) / 2 m D}
$$

where $S(\mathbf{x}, t)=\mathbf{p x}-E t$ and $\rho^{1 / 2}=$ const. Since $\mathbf{u} \sim \nabla \rho=0$, the diffusion momentum vanishes $\mathbf{p}_{u}=0$, as the energy of the corresponding flow $\bar{U}_{u}=0$. As a result, the probability amplitude $\psi(56)$ at each moment is periodically distributed in the space.

For stationary states concentrated in a finite region, in the rest frame of the forces that bind them, or the "center of inertia", we have $S(t)=-E t$ and $\rho(x)$ expresses the localization of the ensemble of particles around this center with $\mathbf{u}^{2}>0$. This indicates that the energy $\bar{U}_{u}$ corresponding to the velocity of the diffusion flow $\mathbf{u}$ is associated with the localization of the particle in the finite region. It is the energy that was transferred to the ensemble of particles at localization in this region (box walls, external force, etc.).

Thus, the quantum potential $\bar{U}_{q}$ appears as the energy that was spent by external forces to localize most of the ensemble of particles in the finite region. If the external force ceases to act, then the diffusion flux will lead to the "spreading" of the ensemble of particles $\rho \rightarrow \rho^{\prime} \sim$ const. Therefore, since diffusion delocalizes the ensemble of particles and, ultimately, leads to $\rho=$ const. and $\mathbf{u}=0$, the energy associated with $\mathbf{p}_{\mathbf{u}}^{2} / 2 m$ represents the potential energy of localization.

As smaller the localization region, as greater the gradient $\rho$ and as greater the localization energy, and this fact explains the uncertainty relations (21).

\subsection{The constancy of light velocity from the constancy of fluctuations}

Diffusion of particles occurs due to their fluctuations in the medium. Therefore, the main characteristic of particle diffusion, expressing the interaction with the medium, is the fluctuation velocity $\mathbf{u}_{q}$. In an ordinary medium, the average of this velocity is zero, and the rms value is nonzero and is proportional to the temperature of the medium:

$$
\overline{\mathbf{u}}_{q}=0, \overline{\mathbf{u}_{f}^{2}} \sim T .
$$


The existence of fluctuations of classical particles in the background field indicates the presence of an effective temperature $T_{V}$ in this field.

In classical statistical mechanics, a fluctuating medium in the form of a gas consisting of atoms and molecules has a rest frame where, for example, a container with gas rests. This frame turns out to be the preferred frame for impurity atoms diffusing in the given gas.

In the case of a background field, the principle of relativity excludes the existence of a preferred inertial frame and requires that fluctuations of classical particles in this vacuum occur identically in all inertial frames.

This is possible only if the particle's mean free path between interactions with the background field is an invariant velocity, which is the same in all inertial frames. This velocity is well known and represents the light velocity $u_{f}=c$.

Thus, the constancy of the light velocity in all inertial frames, property on which the special theory of relativity is based, is a consequence of the principle of constancy of quantum fluctuations in the background field. Moreover, the need for a transition from classical mechanics to relativistic theory also turns out to be one of the consequences of DQM, as well as the special theory of relativity itself.

To the questions: what is the light velocity and why is the light velocity DKM invariant, answers arise: the light velocity is the rate of fluctuation of classical particles in the background field and it is invariant because quantum fluctuations are invariant and the velocity at which the particles fluctuate.

Thus, instead of two postulates about the invariance of quantum fluctuations and the light velocity, in DQM one postulate about the invariance of fluctuations is enough, and the second, about the invariance of the light velocity, is its consequence.

\subsection{The rest energy of a particle as its thermal energy in the background field}

The thermal energy $3 k T / 2$ of particles in a medium (gas, liquid) with temperature $T$ is equal to the average kinetic energy of their thermal fluctuations, i.e. is proportional to the mass and rms velocity of the particles $v_{T}^{2}$ :

$$
\bar{U}_{T}=\frac{3}{2} k T_{V}=m \frac{v_{T}^{2}}{2} .
$$

The same situation, of course, should be for particles of finite mass fluctuating in the background field, i.e. they must have the "thermal" energy of fluctuations proportional to their mass $U_{m} \sim m$.

As already was noted, the localization energy $U_{u}$ is absent for the ensemble of free particles due to $\rho=$ const. Therefore, if we exclude the drift energy also, by choosing the rest frame $\mathbf{v}=0$, then for such free particles the total energy is reduced only to their "thermal" energy $U_{m}$ :

$$
U_{m} \sim m v_{q}^{2}
$$

where $v_{q}$ is the "thermal" velocity of (quantum) fluctuations of the particle.

In conservative diffusion, the thermal velocity $v_{q}$ depends on the intensity of fluctuations of the medium and does not depend on the properties of the fluctuating particle. 
Here $U_{m}$ should be both for localized and free particles, both having a drift velocity and not drifting in a given inertial frame. This part of the particle's energy should have appeared earlier both in theory and in experiments. At interpreting particles in an empty classical space, this energy should have looked mysterious and inexplicable.

The only known part of particle energy with such properties is the rest energy of particles in relativistic theory $E_{0}=m c^{2}$. Therefore, it is precisely it that we can identify with the constant thermal energy of the fluctuations of the particle in the background field $\bar{U}_{m}=E_{0}$. Here, as in the previous Section 5.3, we again come to conclusion that the "thermal" velocity of the quantum fluctuations of the particle in (59) is equal to the speed of light $v_{q} \sim c$.

As the result, the energy of fluctuations of a particle of finite mass in the background field contains a constant part, the energy of "thermal" fluctuations, which manifests itself as the rest energy:

$$
\bar{U}_{m}=m c^{2} .
$$

Thus, if the relativistic theory introduced into physics the rest energy $E_{0}=m c^{2}$, a new addition to the energy of any particle of finite mass, playing a significant role in physics, DQM allows to explain its meaning and origin.

\subsection{Gravitation as a local deficit of fluctuation energy in the background field}

In DQM, the total energy of the system "classical particle + background field" is conserved due to the fact that the particle's fluctuations occur due to the exchange of energy with the background field as well as with any other external field. This means that, as in the case of any other field, an increase (decrease) in the energy of the particle at fluctuation is compensated by a decrease (increase) in the energy of the background field.

As a result, the mean energy of particle's fluctuations transferred to the particle from the background field, increases the total energy of the particle. Accordingly, the total energy of the background field decreases to the same value, and its energy density in the vicinity of the particle becomes less than at a distance.

The influence of one or even many microparticles on the state of the background field is almost unnoticeable. However, when a very large number of particles is concentrated in a sufficiently small region, this effect becomes perceptible in the sense that it already affects fluctuations of particles both in this region and in its vicinity.

A local decrease in the energy density of the background field $\rho_{V}$ in such a region means that the intensity of fluctuations of all particles in this region decreases. Thus, such a local decrease of $\rho_{V}$ is equivalent to local "cooling" of the background field. As a result, an analogue of the thermal diffusion flow of particles arises from regions with a higher $\rho_{V}$ to this region with a lower one, i.e. an area with a very large accumulation of particles effectively attracts other particles.

Due to the conservativity of diffusion, the drift velocity of particle at such a "thermal diffusion" flow will increase cumulatively with each shift and a "thermal diffusion 
acceleration" arises. This new form of acceleration depends only on the local properties of the background field and is practically independent of the masses of accelerated particles.

In a more "cold" region, a decrease in the intensity of fluctuations of particles and "thermal" contractions of the distances between them also occur, i.e. proper times of particles are slowed down, and standard scales are locally contracted.

All these are characteristic properties of gravitation and the fact that they follow from DQM at taking into account the influence of very high concentrations of particles on the energy density of the background field suggests that gravity can be considered as one of the quantum phenomena. Thus, DQM leads to the thermal diffusion treatment of gravitation and implements a natural synthesis of gravity and quantum theory.

As the result, from the two hypotheses of physics - quantum fluctuations and gravity - DQM leaves only the first one as a hypothesis, and turns the second into a consequence of the first, i.e. the theory of gravity appears as a part of DQM. The diffusion treatment of gravitation will be considered in more details in the second paper [12].

\subsection{The scalar field condensate of SM as a candidate to the background field}

The basis of modern particle physics forms the Standard Model (SM). In SM, the mass, and therefore the rest energy, of fundamental particles (three generations of quarks and leptons, gauge bosons $W^{ \pm}, Z^{0}$, and the scalar particle $H^{0}$ ) appears due to the interaction with the scalar field vacuum condensate. The differences in the masses of particles is due to the differences in their interaction constants with the scalar field condensate.

The photon and gluons do not have rest energy due to the fact that they do not directly interact with the scalar field. In terms of DCM, this means that they do not make fluctuations in the sense of chaotic displacements in space and move rectilinearly, because of which their speed is equal to the light velocity. Nevertheless, the field functions associated with them carry out rotational fluctuations, which leads to the quantum behavior of these particles.

In DQM, the same rest energy of SM particles, as discussed above, are related by the thermal energy of their fluctuations in the background field.

This means that the scalar field vacuum condensate of SM can be identified with the background field of DQM. This, in turn, presents new requirements for the condensate itself, namely, vacuum condensate must:

a) fluctuate and these fluctuations must be invariant with respect to the transformations of the Poincare group;

b) interact with classical particles as an external field, acting as the source of their "quantum" fluctuations;

c) the energy transferred to fluctuations of particles as their rest energy should lead to a decrease in the energy of the condensate as a whole to this value.

The main achievement of this treatment is to ensure energy conservation in the system "particle + vacuum condensate" at fluctuations of particles. In particular, a random increase in particle energy at overcoming a potential barrier is compensated by a decrease in the condensate energy to the same value. 
Another positive fact is that a hypothetical background field can now be associated with SM condensate, the presence of which is indirectly confirmed by a large set of experiments in particle physics.

In the second paper [12], where the thermal diffusion in the background field is studied, the connection with the SM condensate and the manifestations of its deformation will be discussed in more detail.

\subsection{Composite models with a diffusion mechanism for small mass}

In SM the number of basic particles, formally introduced as structureless, is large, i.e. several tens. However, SM does not exclude that these particles can have an internal structure at very small distances and this is even desirable.

Firstly, the fundamental nature of the scalar particle is a weakness of SM, since the loop contributions of scalar fields grow with the cutoff energy linearly and become very large long before the Planck scale. Secondly, if the primacy of the stable particles of the first generation of quarks and leptons can still be understood, it is difficult to consider as primary next two generations differing mainly in masses.

In this connection, composite models of SM particles were created, the subparticles of which were called by the initial name preons, using other names for specific models (see, for example, [16]).

However, a mass paradox arose in these models - the smallness of the preon localization region leads to high kinetic energies of preons due to the uncertainty relation, while the observed masses of SM particles are many orders of magnitude smaller. This means that the binding energy of preons should almost completely compensate the contribution of their kinetic energy. But even in this case, the mass difference between the excited states should be much larger than the mass scale of the SM particles, and as a result, the second and third generations of SM fermions cannot simply be the excited states of the first generation.

Attempts to combine the mass paradox with quantum mechanics or to circumvent it in various ways significantly complicated the models, as a result of which the interest to preon models reduced. Instead, there have been developed models with non-composite particles, which generated more problems than they solved and led to even more complex sets of primary particles than even the SM itself.

DQM may reanimate composite SM models, since it has a mechanism for the formation of composite particles of small mass, similar to the mechanism of chemical reactions between impurities [14]. This mechanism can remove the mass paradox, allowing one to avoid the uncertainty relation restrictions.

If in a medium two atoms of impurity began to diffuse at a distance much greater than the mean free path, then the diffusion mechanism prevents their further merging by forming a molecule. For the formation of an ensemble of molecules, it is necessary to localize each of two ensemble of impurity atoms in a certain small volume, and this implies the localization energy as greater, as smaller the final localization volume of these ensembles of atoms. However, if two particles at first were accidentally located at a distance shorter than the mean free path and form a bound state (molecule) within a shorter time, then this compound particle (molecule) diffuses in the same way as other impurity atoms, although with a mass slightly less than the total mass of its atoms.

In [17], it was proposed to use the same mechanism of the formation of composite particles of small mass in DQM and then this mechanism was applied to the simplest of preon models — the rishon model [16]. 
This mechanism, if it turned out to be realistic, would practically remove the mass paradox, making composite models of SM particles consistent and thereby simplifying the situation with further unification of fields.

\section{Conclusion}

In the paper the DCM is formulated as a consistent physical theory starting from the experimentally checked physical principles and leading to the formalism of quantum mechanics as a particular case. The latter was based on the picture of "quantum" particles in empty space spontaneously fluctuating without external source, while in DQM the particles are classical but interact with the fluctuating background field.

DQM is based on two physical principles:

a. The constancy of quantum fluctuations and diffusion: the fluctuations of classical particles in the background field are the same in any inertial frame;

b. The quantum principle of equivalence: the evolution of an ensemble of quantum particles is equivalent to the diffusion of classical particles in the fluctuating background field.

The canonical formalism describing the conservative diffusion, taking into account its specifics, leads to the canonical equations which are non-linear with respect to $\rho$ and $S$. At canonical transformations to the probability amplitude these equations can be linearized and the result is the Schrödinger equation.

DKM thus naturally leads to the formalism of quantum mechanics, including quantum statistics, and also leads to some new consequences. One of them - thermal diffusion in an inhomogeneous background field, which leads to the diffusion treatment of gravity, which will be discussed in the second paperx [12].

The problems for further research are mainly the causes and mechanisms of fluctuations in the background field, as well as their further consequences. The acceptance of these fluctuations as an observational fact in the form of principles of DQM leads to a simple and clear physical picture not only of quantum phenomena, but gravitation too.

\section{Appendix. Interpretation of quantum mechanics in DQ}

\section{A. The derivation of the axioms of quantum mechanics formalism from DQ}

The formalism of quantum mechanics, as mentioned above, founded on a set of mathematical axioms, can now be explained from the point of view of DQM.

The standard list of axioms of quantum mechanics is as follows:

1. For a given physical system, there is a state vector $\Phi$ in the Hilbert space, or a wave function $\psi$ in the coordinate representation, which contains all the information about the system, and the square of the module of the state vector $|\Phi|^{2}$ gives the probability that at a given time the system is characterized by a set of variables characterizing the state of the system.

2. Any observable physical quantity corresponds to a linear Hermitian operator in a Hilbert space.

3. A physical system is an eigenstate of an operator corresponding to an eigenvalue $a$ if $A \Phi_{n}=a_{n} \Phi_{n}$. 
4. An arbitrary wave function, or state vector, of a physical system can be decomposed into a complete orthonormal system of eigenfunctions of a complete set of commuting operators.

5. The result of measuring the observed physical quantity is one of its eigenvalues.

6. The development of a physical system in time is described by the Schrödinger equation, in which the Hamiltonian $H$ is a linear Hermitian operator.

7. Quantum particles with the same properties are indistinguishable.

In DQM, an ensemble of classical particles is described by the functions $\rho, S$, from which the wave function $\psi$ in the coordinate representation is formed. The equations of motion are non-linear for $\rho, S$, but in terms of $\psi, \psi^{*}$, they are linearized and lead to the Schrödinger equation. The mean values of physical quantities in terms of wave functions turn into averages from the corresponding Hermitian operators. The indistinguishability of particles effectively arises from the fact that classical statistics are replaced by quantum statistics due fluctuations between collisions in a condensed media.

All the above postulates are thus the consequences of DQM, a physical picture described by classical physics, but taking into account the specifics of conservative diffusion in a fluctuating background field.

Since the entire formalism of quantum mechanics is derived from the above axioms, then, therefore, this formalism naturally follows from the physical principles of DQM, which consists in describing the fact that particles fluctuate and undergo conservative diffusion not in empty space, but by interacting with the fluctuating background field.

\section{B. Interpretations of quantum mechanics: paradoxes and their solutions in DQM}

The mathematical formalism of quantum mechanics arose in attempts to describe the quantum fluctuations of micro-objects observed in many experiments. But the physical interpretation of this formalism, despite numerous attempts, remained incomplete. For an adequate physical interpretation, it was necessary to determine what is the source of fluctuations of micro-objects - devices, they themselves, or the background surrounding them.

Initially, fluctuations due to devices were studied, since in measurements the influence of macroscopic devices on classical microparticles could systematically introduce an element of randomness. But it turned out that quantum phenomena are not explained by these fluctuations. Firstly, in an ensemble of many similar experiments by a single particle, randomness due to measurements can be reduced to the necessary minimum and, nevertheless, the quantum properties of microparticles, for example, discreteness of energy levels or radioactive decay, do not disappear. Secondly, quantum phenomena determine the structure of matter in the entire universe without any observers and their devices. Thus, it turned out that the devices do not generate, but only record objective quantum fluctuations.

Then, only two possibilities remain for choosing the source of quantum fluctuations - these are either the micro-objects themselves or the background surrounding them. The standard formalism of quantum mechanics was formulated on the basis of the first possibility that the surrounding background is an empty classical space, but micro-objects are "quantum" and they are able to fluctuate spontaneously without any external cause. Note that the assumption of the classical nature of the surrounding background at the microparticle level is purely intuitive and has no observational basis. But the result of this - the randomness of changes in the energy and momentum of microparticles in homogeneous space and time - is paradoxical and caused many objections. 
In classical mechanics, a particle at the bottom of a potential well of finite size and high height remains forever due to a lack of energy required for leaving. In quantum mechanics, with spontaneous fluctuations with a sufficiently high energy, a particle can, in principle, leave this well, overcoming the potential barrier of any finite height and width. If we assume that space is empty and that nothing acts on the particle except the binding potential, then a paradox arises, since the particle has nowhere to take the required (and not small) energy.

Notice that the naive "explanation" of this, that for "quantum" particles there is an uncertainty relation, is a well-known logical trick when the cause is explained by its own consequence. The uncertainty relation describes only one of the consequences of the existence of quantum fluctuations and cannot explain these fluctuations themselves.

An analogue of such a spontaneous fluctuation of energy among people is a paradox, which can be called as the Munchausen paradox. Once, Baron Munchausen, according to him, pulled himself out of the swamp onto the shore with his horse, by strongly pulling himself by his own hair. The paradox is that the baron spoke about this miracle, a respected person who is usually responsible for his statements, but this story, like most miracles, was extraordinary and contradicted the usual experience of other people.

To solve the paradox, it is important that the way that Munchausen pulled himself out of the swamp is not a fact, but only a myth about a wonderful ability. Similarly, although the result of radioactive decay is observed, the statement that the particle that left the potential well gave itself the necessary energy, is not a fact, but the same myth about the miraculous (Munchausen) ability. Note that the desire to maintain an empty surrounding background cannot be a reason for abandoning such a basic law of physics as the conservation of energy of an isolated object.

In DQM, the Munchausen paradox is absent, since the second possibility is used, when the particles remain classical, and the source of their fluctuations is the influence of the background field. When, under the influence of fluctuations of the background field, the particle's energy becomes very large for a short time, the background field energy in this place decreases by the same amount, which ensures the conservation of the total energy of the system as a whole.

Notice that the background field was introduced in some of the treatments, which were discussed above. Although simplified models of fluctuations of this background did not allow these hypotheses to become consistent theories, nevertheless they indicated a way to release of microparticles from spontaneous fluctuations without an external cause by introducing for this a required external source - the fluctuating background field.

The difference between DQM and these simplified models is, firstly, in relying not on particular models, but on physical principles following from experiments and, secondly, in introducing a background field without specifying its specific properties, taking into account only the facts of fluctuations of this field and conserving the total energy of the "particle + background field" system. The third difference is that the principle of relativity requires the invariance of fluctuations and diffusion in this field, which guarantees the conservative nature of diffusion irrespective to its microscopic mechanism.

\section{Slit experiment and the dualism of waves and particles}

In the formalism of quantum mechanics, the question about the physical meaning of the wave-particle dualism was one of the most mysterious. In DQM, where the particles are classical, the concept of particle-wave dualism does not exist for an individual particle. Dualism is a consequence of their interaction with the background field and is manifested only for the ensemble of particles described by the amplitude of probability. 
In a well-known two slit experiment, particles emitted by a source and passing through a screen with two slits form an interference picture on the second screen [11]. In DQM, the particles are not isolated and, in addition to the screen with slits, also interact with the background field along all the path from the source to the second screen. They move along classical, but random in each small section, trajectories corresponding to conservative diffusion, when the motion of an ensemble of particles is described by a complex wave function obeying the Schrödinger equation. When the experiment is repeated many times, an interference picture arises because of the addition not of the probabilities of the two alternatives, but of their probability amplitudes.

The diffusion treatment also provides a simple and satisfactory solution to one of the paradoxes of orthodox quantum mechanics. It was assumed that each particle, at passing through the two slit screen, passes through both slits simultaneously and therefore interferes by itself on the second screen. In reality, this "doubling" of a particle does not follow from the formalism of quantum mechanics and is only a common misconception.

Quantum mechanics, like any statistical theory, gives a probability distribution, in this case determined by the probability amplitude, and does not give any exact predictions for a single random event with an individual particle. And the probability amplitude, like any probability, is determined for a particular ensemble of experiments on individual particles. The possibility of the passage of individual particles through one of the two slits determines a certain probability distribution and interference refers to a specific probability distribution due to the addition of the amplitudes of these two alternatives. This concerns a formal objection to the above misconception.

Thus, if, as it was before, we say about particles and screens in the empty classical space, then there is no way to explain such a non-trivial phenomenon as interference in the probability distribution for particles passed through only one of the slits. The use of such an absurd "explanation" as the simultaneous (!) passage of a particle through two slits is a consequence of the absurdity of the initial assumption that the fluctuating (!) particles are isolated.

DQM, on the other hand, allows to give a consistent physical explanation of this experiment, taking into account, in addition to interaction with screens, the interaction of particles with a background field, after which the mystery of the situation disappears. In this picture, neither particles nor screens are responsible for the interference picture, since it is due to the specificity of diffusion in that non-trivial, i.e. fluctuating background field with which particles interact before and after passing one of the slits.

This process occurs without interference when only one of the slits is open, and occurs with interference when both slits are open. Each particle, of course, passes only through one of the slits and "does not know" whether the second slit is open or not. But the background field "knows", through globally defined probability amplitudes describing diffusion in this field, each slit is open or closed. Therefore, an ensemble of particles corresponding to repeated experiments with a single particle either interferes on the second screen or not depending on what probability distribution is formed upon conservative diffusion in the background field with one or another configuration of slits and screens.

Thus, it is not a single particle that interferes with itself, but an ensemble of particles in the fluctuating background, i.e. the interference picture is formed by the ensemble of experiments only. The fact that a particle has a low probability of falling into certain parts of the screen and a high probability in others means only the nontrivial probability distribution due to the addition of amplitudes. This, in turn, is evidence of the presence, in addition to particles and the "device" (screens), of a background field, the fluctuations in which give such a nontrivial distribution. 
And, finally, the disappearance of interference at "peeping" over which of the two open slits each particle passed through was previously presented as a paradoxical fact. In DQM, this phenomenon is not a paradox, because when there is no "peeping" over the particles, the ensemble is clean and the amplitudes are summed, and if the particles, in addition to the screen with slits and the background field, interact with "counting" devices behind the slits, then such an ensemble will become mixed and summed the probabilities of two alternatives.

\section{References}

1. Madelung, E. (1926) Zs. Phys., 40, 322.

2. Ehrenfest, P. (1927) Zs. Phys., 45, 455.

3. Schrödinger E. (1930) Sitz. Preus. Akad. Wiss., 418; (1932) Ann. I.H.P. 2(4) 269.

4. Fürth, R. (1933) Zs. Phys., 81, 143.

5. Fényes, I. (1952). Z. Phys., 132, 81.

6. Nelson E. (1966) Phys. Rev., 150, 1057; (1985) Quantum Fluctuations. Pr.U.P.

7. Guerra F., Marra R. (1983) Phys. Rev. D 28, 1916.

8. Weizel (1953) Zs. Phys., 135, 270.

9. Grabert H., Hänggi P., Talkner P. (1979) Phys. Rev. A 19, 2440.

10. Feynman R. P. (1948) Phys. Rev., 20(2), 367.

11. Feynman R. P., Hibbs A. R. (1965) Quantum Mech. and Path Integrals. McG.-H.

12. Zakir, Z. (2020) Quant. and Grav. Phys., 1:004-7129.

13. Zakir, Z. (2020) Diffusion Quantum Mechanics and Diffusion Gravity. CTPA, T.

14. Landau L. D., Lifshitz E. M. (1980) Statistical Physics. 1. Perg. P.

15. Tersoff J., Bayer D. (1983) Phys. Rev. Lett. 50, 553.

16. Harari H. (1979) Phys. Lett. 86B, 83; Shupe M.A. (1979) Phys. Lett. 86B, 87.

17. Zakir, Z. (2016) Theor. Phys., Astroph. and Cosmol. 11, 1. 